\title{
ENTIERE IDENTITIES BETWEEN OPHIOCOMINA NIGRA IGKAPPA GENE AND HUMAN IMMUNOGLOBULIN KAPPA LOCUS.NEW ASPECTS OF INVERTEBRATE IGKAPPA GENES
} (IPA)

\author{
Michel Leclerc \\ Immunology of Invertebrates \\ Department of Cell Biology, Developmental Biology, Immunology \\ University of Orleans, France \\ E-mail: mleclerc45@gmail.com
}

\begin{abstract}
Entiere identities between Invertebrate Ophiocomina nigra IGKappa gene and Human IGK gene are confirmed, in the present work, at the level of immunoglobulin domains (constant and variable). The transcriptome of the Ophuirid: Ophiocomina nigra IGKappa gene was discovered recently(1). Since it was synthesized de novo and cloned in a pUC-GW-Kan plasmid (2) which was a gift of Bo Huang laboratories. The original sequence of the IGKappa gene, after cloning, was the following in 5'-3':
\end{abstract}

\section{ORIGINAL SEQUENCE}

GAGGAACTGCTCAGTTAGGACCCAGACGGAACCATGGAAGCCCCAGCGCAGCTTCT СTTCCTCCTGCTACTCTGGCTCCCAGATACCACTGGAGAAATAGTGATGACGCAGTCT CCAGCCACCCTGTCTGTGTCTCCAGGGGAAAGAGCCACCCTCTCCTGCAGGGCCAGT CAGAGTGTTACCAGCAACTTAGCCTGGTACCAGCAGACACCTGGGCAGTCTCCCAGG CTCGTCATCTATGGTGCATCCAGCAGGGCCAGTGGTGTCCCAGCCAGGTTCAGTGGC AGTGGGTCTGGGACAGAGTTCACTCTCACCATCAGCAGCCTGCAGTCTGAAGATTTT GCAGTTTATTACTGTCAGCAGTATAATAAGTGGCCGCACACTTTTGGCCAGGGGACCA AGCTGGACATCAAACGAACTGTGGCTGCACCATCTGTCTTCATCTTCCCGCCATCTGA TGAGCAGTTGAAATCTGGAACTGCCTCTGTTGTGTGCCTGCTGAATAACTTCTATCCC AGGGAGGCCAAAGTACAGTGGAAGGTGGATAACGCCCTCCAATCGGGTAACTCCCA GGAGAGTGTCACAGAGCAGGACAGCAAGGACAGCACCTACAGCCTCAGCAGCACCC TGACGCTGAGCAAAGCAGACTACGAGAAACACAAAGTCTACGCCTGCGAAGTCACC CATCAGGGCCTGAGCTCGCCCGTCACAAAGAGCTTCAACAGGGGAGAGTGTTAGAG GGAGAAGTGCCCCCACCTGCTCCTCAGTTCCAGCCTGACCCCCTCCCATCCTTTGGCC TCTGACCCTTTTTCCACAGGGGACCTACCCCTATTGCGGTCCTCCAGCTCATCTTTCAC CTCACCCCCCTCCTCCTCCTTGGCTTTAATTATGCTAATGTTGGAGGAGAATGAATAAA TAAAGTGAATCTTTGCAAAAAAAAAAAAAAAAAAAAAAAAAAAAAAAAAAAAAAA AAAAAAAAAAAAAAAAAAAAAAAAAAAAAAAAAAAAAAAAAAAAAAAA

The original gene, the original protein issued from this last one share total identity with Homo 
sapiens immunoglobulin kappa locus, mRNA (cDNA clone MGC:22645 IMAGE:4700961) : they have a complete identity(Fig1)

The Sequence of the concerned gene is ID: BC030813.1

At last the Protein GenBank (3) has the following number: AAH30813.1 with 234 amino acids as shown below:

MEAPAQLLFLLLLWLPDTTGEIVMTQSPATLSVSPGERATLSCRASQSVTSNLAWYQQTP GQSPRLVIYGASSRASGVPARFSGSGSGTEFTLTISSLQSEDFAVYYCQQYNKWPHTFGQG TKLDIKRTVAAPSVFIFPPSDEQLKSGTASVVCLLNNFYPREAKVQW KVDNALQSGNSQESVTEQDSKDSTYSLSSTLTLSKADYEKHKVYACEVTHQGLSSPVTK SFNRGEC

It is shown, for the first time that an invertebrate IGKappa gene shares entiere identity with a human immunoglobulin (Figure 1)

A similar aspect was obtained with the sea star: Asterias rubens (4) (Asterids, Echinodermata) which presented 2 IG sites.

\section{REFERENCES}

Leclerc, M., Marie, Y., Davoult, D., Jolly, A., de la Grange, P., \& Paris, I. G. (2018). A true new gene in ophiocomina nigra: an ophuirid Igkappa gene. J Appl Biotechnol Bioeng, 5(1), $17-18$.

Leclerc, M. (2021). Immunol. Lett. Submitted

Hutchinson, A. T., Jones, D. R., Winter, P. M., Tangye, S. G., \& Raison, R. L. (2014). Cell membrane associated free kappa light chains are found on a subset of tonsil and in vitroderived plasmablasts. Human immunology, 75(9), 986-990.

Vincent, $\mathrm{N}$ et al. (2014). Meta Gene 2, 320-22 


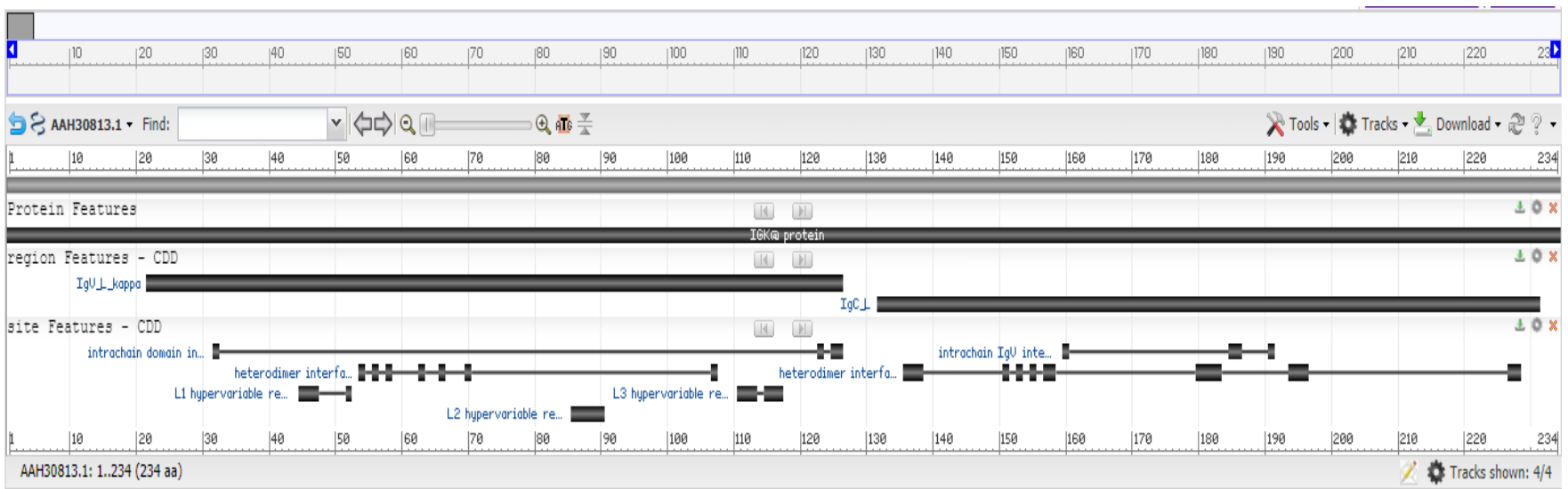

Figure 1:

IGK@ protein [Homo sapiens] graphic (in dark) by NCBI (https://www.ncbi.nlm.nih.gov/protein/AAH30813.1?report=graph) shares IG domains with Ophiocomina nigra IGKappa protein(in grey) issued from ophuirid IGKappa gene

GenBank: AAH30813.1 protein issued from IGK gene has two immunoglobulin domains:

1. Region 1

Region : IgV_L_kappa

Comment : Immunoglobulin (Ig) light chain, kappa type, Variable (V) domain

Location : $22 \ldots 126$

Length 105 aa

1. Region 2

Region : IgC_L

Comment : Immunoglobulin constant domain

Location : $132 \ldots 231$

Length 100 aa

\section{Copyrights}

Copyright for this article is retained by the author(s), with first publication rights granted to the journal. This is an open-access article distributed under the terms and conditions of the Creative Commons Attribution license (http://creativecommons.org/licenses/by/4.0/). 